\title{
Likelihood ridges and multimodality in population growth rate models
}

\author{
Leo Polansky ${ }^{1,5}$ Perry de Valpine, ${ }^{1}$ James O. Lloyd-Smith, ${ }^{2,3}$ and Wayne M. Getz ${ }^{1,4}$ \\ ${ }^{1}$ Department of Environmental Science, Policy, and Management, University of California, 137 Mulford Hall, \\ Berkeley, California 94720-3112 USA \\ ${ }^{2}$ Center for Infectious Disease Dynamics, Pennsylvania State University, University Park, Pennsylvania 16802 USA \\ ${ }^{3}$ Department of Ecology and Evolutionary Biology, University of California, Los Angeles, California 90095-1606 USA \\ ${ }^{4}$ Mammal Research Institute, University of Pretoria, Pretoria, South Africa
}

\begin{abstract}
A central problem in population ecology is to use time series data to estimate the form of density dependence in the per capita growth rate (pgr). This is often accomplished with phenomenological models such as the theta-Ricker or generalized Beverton-Holt. Using the theta-Ricker model as a simple but flexible description of density dependence, we apply theory and simulations to show how multimodality and ridges in the likelihood surface can emerge even in the absence of model misspecification or observation error. The message for model fitting of real data is to consider the likelihood surface in detail, check whether the bestfit model is located on a likelihood ridge and, if so, evaluate predictive differences of biologically plausible models along the ridge. We present a detailed analysis of a focal data set showing how multimodality and ridges emerge in practice for fits of several parametric models, including a state-space model with explicit accommodation of observation error. Best-fit models for these data are biologically dubious beyond the range of the data, and likelihood ratio confidence regions include a wide range of more biologically plausible models. We demonstrate the broad relevance of these findings by presenting analyses of 25 additional data sets spanning a wide range of taxa. The results here are relevant to information-theoretic and Bayesian methods, which also rely on likelihoods. Beyond presentation of best-fit models and confidence regions around individual parameters, effort toward understanding features of the likelihood surface will help ensure the most robust translation from statistical analysis to biological interpretation.
\end{abstract}

Key words: Accipiter nisus; biological plausibility; density dependence; generalized Beverton-Holt; per capita growth rate; Sparrowhawk; state-space models; theta-Ricker; time series analysis.

\section{INTRODUCTION}

Determining the effect of density on the per capita growth rate (pgr) $\log _{e}\left(N_{t+1} / N_{t}\right)$, where $N_{t}$ is the density of the population at time $t$, has critical implications for population ecology theory (May and Oster 1976), conservation biology (Ginzburg et al. 1990, Sæther et al. 2000), and resource management (Getz and Haight 1989, Sæther et al. 1996, Myers et al. 1999, Aanes et al. 2002). One theoretical prediction about the form of the pgr suggests that it is sigmoidal over a broad range of densities, and convex upward for values near and below the expected density (Getz 1996). The recent availability of relatively long-term data time series has allowed tests of such theory. Notable among these is the report of Sibly et al. (2005) on fitting the theta-Ricker model to 1780 time series from the Global Population Dynamics Database (GPDD) (maintained by the National Environment Research Council [NERC] Center for Popula-

Manuscript received 6 August 2008; revised 16 October 2008; accepted 13 November 2008. Corresponding Editor: E. G. Cooch.

${ }^{5}$ E-mail: leop@nature.berkeley.edu tion Biology). They conclude that the effects of density on the pgr are often concave, implying density dependence sets in most strongly well below the long-term expected density for a majority of species. If true, this finding has dramatic implications for wildlife management and conservation, as well as for fundamental ecological theory (Reynolds and Freckleton 2005).

There have been many theoretical models of the pgr motivated by natural systems that include, for example, the effects of age structure (Ellner and Turchin 1995), Allee effects, or multiple species (Turchin 2003). Omission of such effects has recognized consequences leading to erroneous conclusions regarding the role of density dependence in population dynamics (Turchin 1990, Wolda and Dennis 1993). For many species, however, such omissions are appropriate: fitting relatively simple models is often a first step when assessing the dynamic properties of the density-dependent feedback on population growth and a means for obtaining a model that can be used to predict the population growth response at different population densities (Dennis and Taper 1994).

In this paper, we emphasize the importance of thoroughly investigating the likelihood surface produced 

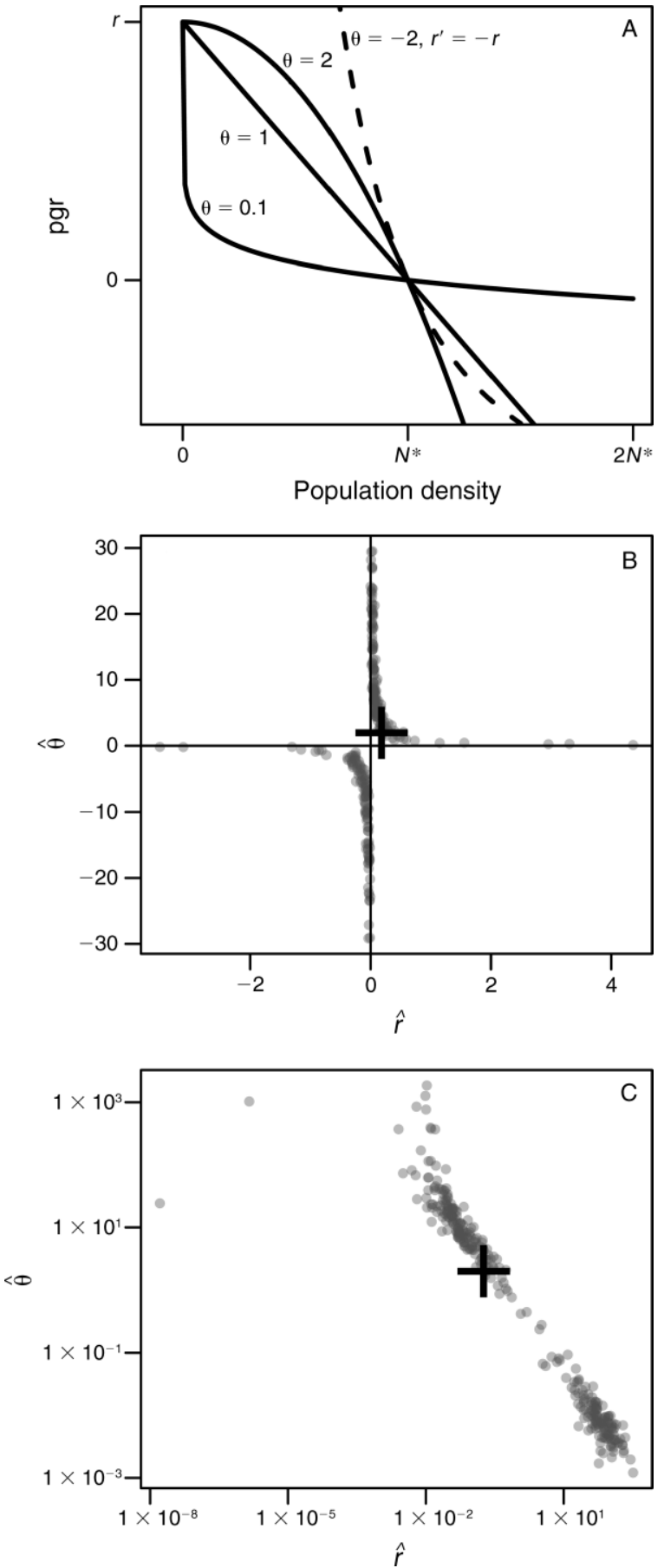

FIG. 1. (A) Theoretical per capita growth rate (pgr) curves given by the theta-Ricker model for different values of $\theta$ and a specific but arbitrary $r>0(r$ is the maximum of a pgr that declines to 0 as population density increases to $K>0 ; \theta$ describes the form of the density dependence). The curve associated with the negative value of $\theta$ uses $r^{\prime}=-r$. (B, C) Gray points show ML (maximum likelihood) estimates $(\hat{r}, \hat{\theta})$ from a theta-Ricker model fit to 300 simulated stationary time series of length 20 generated by the theta-Ricker with process noise (TRPN) model, with generating parameters $r=\log _{e}(1.2)$ and $\theta$ $=2$ located at the plus sign. In (B), estimates are allowed to take on any value, and for clarity $247(82 \%)$ of these pairs are from fitting density-dependent pgr models. Using the theta-Ricker model as a simple but flexible description of density dependence, we use theory and simulations to show how multimodality and ridges in the likelihood surface can emerge even in the absence of model misspecification or observation error. Our analysis indicates that when fitting models to empirical data, one should consider the likelihood surface in detail, check for ridges containing the best-fit model, and, if they exist, evaluate predictive differences of other biologically plausible and statistically supported models along these ridges. We illustrate these ideas with several different pgr models to an Accipiter nisus (Sparrowhawk) time series obtained from the GPDD; the general issue of multimodality and ridges is shown to exist in 25 additional data sets spanning a wide range of taxa. We discuss the implications of our results for biological inference, including model selection and Bayesian methods, and for the claim by Sibly et al. (2005) that many populations exhibit "a strongly concave relationship between a population's growth rate and its size."

\section{Stochastic pgr Models and Parameter Estimation}

The framework we use for estimating the parameters of a pgr model, $g(N)$, is the prediction equation $\log _{e}\left(N_{t+1}\right)=\log _{\mathrm{e}}\left(N_{t}\right)+g\left(N_{t}\right)+\mathrm{v}_{t}$, where the process noise $v_{t}$ is assumed to be a sequence of independent, identically distributed (iid) values drawn from a normal distribution (e.g., Dennis and Taper 1994) with zero mean and standard deviation $\sigma_{\mathrm{p}}$. Accounting for observation error in addition to process noise with real density time series can be informative (de Valpine and Hastings 2002, de Valpine and Hilborn 2005, Dennis et al. 2006, Sæther et al. 2007, Lillegard et al. 2008), and we accommodate this level of stochasticity using a statespace model (Eq. A.4) with an iid normal random variable $\varepsilon_{t}$ with zero mean and standard deviation $\sigma_{\mathrm{o}}$ for observation error (see Appendix: sections A2 and A4). Formulations of all models (likelihood functions) used in a maximum likelihood (ML) analysis to obtain bestfit parameter estimates and construct confidence regions are given in the Appendix (Eqs. A.1-A.4). Throughout, we denote the vector of model parameters by $\boldsymbol{\Theta}$, the maximum likelihood estimated parameters by $\hat{\boldsymbol{\Theta}}$, and the value of the log-likelihood function at $\boldsymbol{\Theta}$ by $\ell(\boldsymbol{\Theta})$.

Consider the log-transformed theta-Ricker pgr model (Thomas et al. 1980) $g\left(N_{t}\right)=r\left(1-\left(N_{t} / K\right)^{\theta}\right)$, where $r$ is the maximum of a pgr that declines to 0 as population density increases to $K>0$ (where $K$ is the population density for which the pgr is zero) and $\theta$ describes the form of the density dependence (Fig. 1A). Fixing $\theta=1$

shown, with the remaining pairs lying along the axes outside the boundaries of the graph. In (C), the $r$ and $\theta$ estimates are restricted to be positive, and a $\log -\log$ scale is used. However, the hyperbolic-shaped ridge (negative linear shape on a log-log scale) of similar likelihood values remains. 
produces the widely used Ricker model. Fig. 1A shows how different regions of the $r-\theta$ parameter space describe comparable pgr models near the point equilibrium density $N_{t}=N^{*}=K$ for all $t$ : those with $\theta<1$ and $\theta>1$ being described as concave and convex, respectively. Since the slope of these curves at the fixed-point equilibrium density $N^{*}$ is $-r \theta / K, \Theta$ values in the first or third quadrant of the $r-\theta$ plane could be expected to define models that fit the same data, if clustered around $N^{*}$, comparably well. Statistical theory does not distinguish the realism of the first or third quadrants in the $r-\theta$ plane, providing only that one of the modes will be the asymptotically correct estimate (Severini 2000, Dennis et al. 2006). We focus only on interior modes in the likelihood surface, those that are away from the $\Theta$ boundaries $\sigma_{\mathrm{p}}=0$ and $\sigma_{\mathrm{o}}=0$. For the theta-Ricker model, a simple solution to prevent multimodality in the likelihood function is to bound estimates of $\Theta$ to the first quadrant of the $r-\theta$ plane using phenomenological arguments to reject a concave pgr (Getz 1996), since these imply the strongest levels of density dependence occur at the lowest densities rather than at some critical density as the competitive impact among individuals becomes particularly intense (e.g., when the total area of a relatively homogeneous region divided by the number of individuals in the region has a value less than the maximum area an individual requires to meet all its needs, then the inverse of this is the critical density).

We conducted a simulation experiment to quantify the distribution of point estimates for the theta-Ricker model by generating 300 stationary time series of length 20 (a generous sample size relative to many time series in the GPDD; see the Appendix) using the stochastic logtransformed theta-Ricker with process noise (TRPN) model with parameter values $r=\log _{e}(1.2)=0.18, \theta=2$, $K=1$, and $\sigma_{\mathrm{p}}=0.05$. The deterministic trajectories defined by the model at these parameters except with $\sigma_{\mathrm{p}}$ $=0$ are stable and constant. Allowing $r$ and $\theta$ to assume any values, the best-fit TRPN model (Eq. A.1) fit to each of the time series found ML estimates of $\Theta$ in both the first and third quadrants of the $r-\theta$ plane, with $44.3 \%$ of them lying in the third (implausible) quadrant and an additional $3 \%$ in the first quadrant also with concave $(0<\hat{\theta}<1)$ estimates of the pgr (Fig. 1B). Because of the modeled pgr increase toward infinite as $N$ goes to zero for negative values of $r$ and $\theta$ (Fig. 1, Appendix: section A1), we applied biological rationale to constrain $r$ and $\theta$ to positive values. Reanalyzing these same time series with these constraints results in $\hat{\theta} \leq 1$ for $42 \%$ of the time series, with negatively correlated values of $\hat{r}$ (Fig. 1C). Even in the absence of any process model misspecification, observation error, or incorrect treatment of stochastic effects, the best-fit model often dramatically incorrectly estimates the shape of the pgr for the generating parameters considered here. The distribution of the point estimates in Fig. 1B,C illustrates a subset of parameter space that could provide similar likelihood values to the global maximum, given a particular population abundance time series. We note that other generating parameters producing deterministic oscillations in $N_{t}$ would provide much more accurate point estimates (Schaffer et al. 1986, Kendall 2001, Polansky et al. 2008), but the absence of density-dependent induced oscillations may be quite widespread (Sibly et al. 2007, but see our Discussion), and is relevant for the GPDD data analyzed here.

Before moving to some case studies, we discuss two additional relevant issues besides observation error. First, rather than multiple time series, typically one has a single time series from which to establish confidence in the best-fit model. Towards this end, we can construct a joint profile likelihood surface, a general model diagnostic tool proving useful in at least several density-dependent investigations (Hilborn and Mangel 1997, de Valpine and Hilborn 2005, Dennis et al. 2006), for the parameters controlling the important features of the pgr model. We use the theta-Ricker model to illustrate this procedure. By fixing $\psi_{0}=\left(r_{0}, \theta_{0}\right)$ at points over a grid of values in the $r-\theta$ plane, and then maximizing the log-likelihood function in the remaining parameter dimensions of $\Theta$ to obtain the maximum loglikelihood value $\ell\left(\hat{\Theta}_{\psi_{0}}\right)$ at each point in the grid, we obtain a surface of joint profile log-likelihood values $\ell\left(\hat{\Theta}_{\psi_{0}}\right)$ over the grid. By transforming this surface to $\Lambda_{\psi_{0}}$ $=2\left[\ell(\hat{\Theta})-\ell\left(\hat{\Theta}_{\psi_{0}}\right)\right]$, we can use the likelihood ratio test (LRT) to draw contour levels at quantiles from a chisquare distribution $\chi_{m}^{2}$, where $m$ is the difference between the number of parameters in $\Theta$ and $\boldsymbol{\psi}_{0}$, which will depict the approximate boundaries for alternative models that cannot be rejected at the corresponding $P$ value (Meeker and Escobar 1995). We choose this method to estimate confidence intervals, rather than bootstrapping (Shumway and Stoffer 2000, Dennis et al. 2006) or data cloning (Lele et al. 2007), other available frequentist based methods for estimating confidence in parameter estimates of state-space models, because it both describes the likelihood surface geometry and represents a parsimonious approach to the relatively simple models that include only one type of stochasticity; a side-by-side comparison of the different methods of statistical inference under different levels of model complexity and types of data could be a potentially illuminating topic of further research.

Second, a priori insistence on any one parametric model family is not always justified. Motivated by the point estimation challenges facing the TRPN model and with the knowledge that in some cases predictions may depend on the choice of phenomenological model (Wood and Thomas 1999), we also consider the log-transformed Beverton-Holt model $g\left(N_{t}\right)=r-\log _{e}\left(1+\left(N_{t} / K\right)\right)$ and its generalization $g\left(N_{t}\right)=r-\log _{e}\left(1+\left(N_{t} / K\right)^{\gamma}\right)$ (where $K$ is the population density at which the effects of density dependence are maximized) with the added shape parameter $\gamma$ (which controls the form of density 
dependence) as an appropriate alternative model family (Maynard Smith and Slatkin 1973, Bellows 1981, Getz 1996, Myers et al. 1999). Here, $r$ is as above, $\gamma$ generalizes the density-dependent pgr curve to allow both concave and sigmoidal shapes, and the point equilibrium density is at $N^{*}=K\left(e^{r}-1\right)^{1 / \gamma}$. The log-transformed generalized Beverton-Holt with process noise $(\gamma$-BHPN; Eq. A.2) model is given by $\ln \left(N_{t+1}\right)=\ln \left(N_{t}\right)+r-\ln \left(1+\left(N_{t} / K\right)^{\gamma}\right)+$ $v_{t}$. Although multimodality in the likelihood surface for this model is expected to be rare (Appendix: section A1), lack of information about $r$ can also result in wildly inaccurate best-fit pgr models (Polansky et al. 2008). Finally, before moving on to our real data case studies, we note that we use the abbreviation ALT to denote alternative parameters chosen somewhat arbitrarily to illustrate biologically plausible models that are statistically indistinguishable from best-fit parameters.

\section{Case Studies}

We first analyze an extensively studied $A$. nisus population time series from the GPDD for which density-dependent effects have previously been established (Newton and Marquiss 1986, Newton and Rothery 1997). Two separate estimates of the shape parameter with a TRPN model have been published for this population: Sæther et al. (2002) reported $\hat{\theta}=2.57\left(\sigma_{\mathrm{p}}=\right.$ \pm 0.69 ) using ML methods, with $\hat{r}$ estimated independently (data: 1975-1989), while Sibly et al. (2005) reported $\hat{\theta}=-3.16((-12.6,10)$ estimated $95 \%$ confidence interval) using least-squares fitting on a grid of values (data: 1972-1989). Here we focus on the TRPN model as the starting place for a density-dependent investigation, using the full data (1972-1989) available from the GPDD (see Appendix: sections A2 and A4 for details on additional analyses and other available data for this population).

All three models, TRPN, $\gamma$-BHPN, and theta-Ricker state-space (TRSS), present joint profile likelihood surfaces with extended ridges containing their best-fit parameter estimates (Fig. 2A, C, E; Table 1). For both the TRPN and TRSS models, likelihood surfaces are also multimodal, with the global maximum occurring in the third quadrant of the $r-\theta$ plane (Table 1, Fig. 2A, C; Fig. A3). The TRSS joint profile likelihood surface (Fig. 2C) confirms that the multimodality and ridges are not driven by a failure to accommodate observation error (differences in how likelihood values are calculated between the state-space model and the other models prohibits comparing these values directly across models; see Appendix: sections A2 and A4). Despite the single mode in the likelihood surface of the $\gamma$-BHPN model, it does not appear to reduce the range of statistically plausible pgr models.

Global models using theta-Ricker descriptions of density dependence yield unreasonable best-fit estimates of $r$ (Table 1) and subsequent model predictions for population trajectories when initial densities are low (Table 2). We used Fig. 2A, C, E as guides to identify
ALT models that are both biologically and statistically plausible, comparing likelihood differences using a LRT (Tables 1 and 2). Fig. 2B, D, F illustrates how the bestfit and ALT models exhibit similar behavior over the range of the data but pronounced differences at low population densities; examination of residuals fails to reveal any evidence of model misspecification by either best-fit or ALT models (Fig. A2). ALT models predict substantially slower population recoveries than any of the related best-fit models, as indicated by the positive skewness of predicted values of $\mathrm{N}$ in contrast with the negative sample skews of best-fit models for these scenarios (Table 2).

Given that likelihood ridges are common to all generalized models considered here, we explored the broader applicability of our findings by fitting the TRPN model to 25 additional time series for which concave pgr has been reported (Sibly et al. 2005), including three mammal, 10 bird, four fish, and eight insect species. From the best-fit mode in the first quadrant of the $r-\theta$ plane, we located along the joint profile likelihood ridge other supported values of $r$ and $\theta$. Fig. 3 summarizes these results, showing that for each concave best-fit pgr curve there is a substantially different convex ALT model which cannot be rejected statistically at the conventional $P$ value of 0.05 , and usually the $P$ values are on the order of 0.3-0.5 (Appendix: Table A4, Fig. A3). Included in these additional data sets is a time series for Columba oenas, which has been analyzed by Ward (2006) using a theta-Ricker model with observation error and a Bayesian approach to parameter estimation. For this data set, using either process noise only or observation error only models the frequentist $0.95 r-\theta$ confidence region determined by the LRT defines a ridge in the $r-\theta$ plane containing the best-fit model (Fig. A5), matching by eye the $95 \%$ central posterior density interval of these parameters in Fig. 1 of Ward (2006).

\section{Discussion}

For general models of density dependence, the ML point estimates derived from many simulated and real data sets are essentially uninformative about the pgr at densities where the flexibility of a generalized pgr model results in substantial differences from a restricted model. Thus, as in the case of linear and nonlinear regression, prediction outside the range of observed data is ill advised (but see Myers et al. [1999] for a study where such prediction using linear models may be warranted). Although the "true" form of the pgr of any population may be restricted to the environmental context and location from which the data were obtained (Krebs 2002), without confident pgr estimates at low densities resulting in, e.g., elliptical contour levels around a single maximum in the joint profile likelihood surface of interest, the point estimates (corresponding to concave pgr curves) for the time series analyzed here offer little insight to the nature of the "true" pgr curves beyond the range of observed data. 

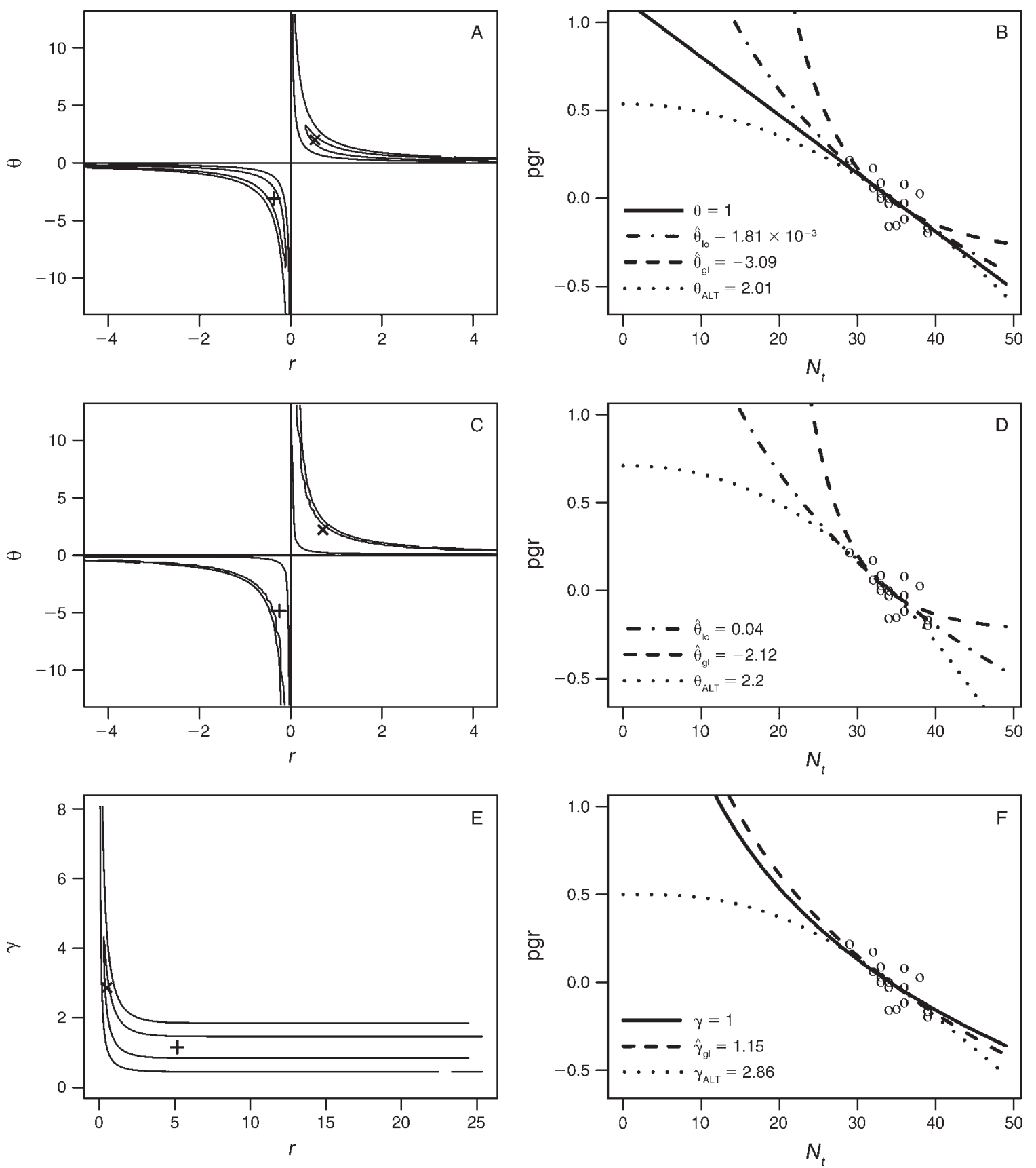

FIG. 2. Joint profile likelihood surfaces of the parameters describing the pgr curve of the Accipiter nisus data set and the corresponding predicted pgr curves for the flexible pgr models of Table 1: (A, B) the theta-Ricker process noise (TRPN) model; $(\mathrm{C}, \mathrm{D})$ the theta-Ricker state-space (TRSS) model; (E, F) the generalized Beverton-Holt process noise $(\gamma$-BHPN) model. The joint profile $\log$-likelihood values $\ell\left(\hat{\Theta}_{\psi_{0}}\right)$ have been transformed to $2\left[\ell(\hat{\Theta})-\ell\left(\hat{\Theta}_{\psi_{0}}\right)\right]$; for models with theta-Ricker type density dependence we used the log-likelihood value from the mode in the first quadrant of the $r-\theta$ plane. Global best-fit (gl) parameters are located at plus signs, and ALT locations are at crosses. Local first-quadrant (lo) best-fit parameters for theta-Ricker type density-dependent models lie beyond the $x$-axis limits in (A) and (B). Contour lines are at the 0.5 and 0.95 quantiles of a $\chi_{2}^{2}$ distribution, corresponding to the closest and farthest lines from plus signs, respectively; for the TRSS model, the $r-\theta$ axes are within the $95 \%$ confidence region. The data $\left[\log _{e}\left(N_{t+1} / N_{t}\right)\right.$ vs. $\left.N_{t}\right]$ are shown by circles.

In summary, there are two specific points from this research. First, as widely recognized, careful study of the estimation properties of models using simulated data can be an invaluable exercise before moving on to em- pirical data. Second, although potentially computationally expensive, calculating joint profile likelihood surfaces is a useful diagnostic tool for obtaining greater understanding from broad confidence intervals. While 
TABLE 1. Results of different density-dependent models fit to the Accipiter nisus data set.

\begin{tabular}{|c|c|c|c|c|c|c|c|}
\hline Model and likelihood location & $r$ & $N^{*}$ & $\theta, \gamma$ & $\sigma_{\mathrm{p}}$ & $\sigma_{\mathrm{o}}$ & $\ell(\Theta)$ & $\overline{\text { LRT } P}$ \\
\hline RPN, best fit & 1.13 & 34.30 & & 0.08 & & 18.61 & $0.54(1)$ \\
\hline TRPN, first quadrant best fit & 635 & 34.20 & 0.00181 & 0.08 & & 18.79 & \\
\hline TRPN, third quadrant best fit & -0.37 & 34.41 & -3.09 & 0.08 & & 19.07 & \\
\hline TRPN, ALT & 0.54 & 34.38 & 2.01 & 0.08 & & 18.38 & $0.50(2)$ \\
\hline TRSS, interior first quadrant best fit & 31.44 & 34.19 & 0.04 & 0.04 & 0.07 & 18.41 & \\
\hline TRSS, interior third quadrant best fit & -0.25 & 33.98 & -4.83 & 0.05 & 0.06 & 18.49 & \\
\hline TRSS, interior ALT & 0.71 & 34.23 & 2.2 & 0.03 & 0.07 & 18.30 & $0.89(2)$ \\
\hline BHPN, best fit & 9.15 & 34.18 & & 0.08 & & 18.63 & $0.57(1)$ \\
\hline$\gamma$-BHPN, best fit & 5.17 & 34.21 & 1.15 & 0.08 & & 18.79 & \\
\hline$\gamma$-BHPN, ALT & 0.50 & 34.37 & 2.86 & 0.08 & & 18.44 & $0.71(2)$ \\
\hline
\end{tabular}

Notes: Estimates of fixed-point equilibrium density $N^{*}$, rather than $K$, are provided for comparison between theta-Ricker (TR) and Beverton-Holt (BH) types of density dependence (for the Ricker and theta-logistic models, $K$ is the population density for which the pgr is zero; for the Beverton-Holt and generalized Beverton-Holt models, $K$ is the population density at which the effects of density dependence are maximized). Values of $r, \theta$, and $\gamma$ for the biologically plausible ALT models are chosen, not estimated ( $r$ is the maximum of a pgr [per capita growth rate] that declines to 0 as population density increases to $K>0 ; \theta$ describes the form of the density dependence; $\gamma$ is the parameter controlling the form of density dependence in the generalized Beverton-Holt model). Likelihood ratio test (LRT) $P$ values are used to compare the support of the first quadrant best-fit models against simpler process $(\theta=1$ or $\gamma=1)$ or ALT models within family (process and stochastic structure), where the LRT statistic follows a $\chi_{m}^{2}$ with the degrees of freedom $m$ given in parentheses. Other variables are: $\sigma_{\mathrm{p}}$, process noise standard deviation; $\sigma_{\mathrm{o}}$, standard deviation for observation error; and $\ell(\Theta)$, the maximum log-likelihood value. Model abbreviations are: RPN, Ricker $(\theta=1)$ with process noise; TRPN, thetaRicker with process noise; TRSS, theta-Ricker with process noise and observation error; BHPN, Beverton-Holt $(\gamma=1)$ with process noise; $\gamma$-BHPN, generalized Beverton-Holt with process noise. Empty cells are those in which values are not estimated (because the model does not contain that particular parameter) or computed (because it does not make sense to compare models with biologically implausible parameters, i.e., best-fit models in the third quadrant of the $r-\theta$ plane).

obtaining best-fit parameter estimates is a natural first step in evaluating a model given data, a critical eye must be kept on the biological plausibility of these models. Using the full range of analytical tools, statistics can tell us when a model is useful, and when it is not.

A direct consequence of this research regards the conclusion by Sibly et al. (2005), based upon point estimates of the TRPN $\theta$ parameter allowed to take on positive or negative values, that the relationship between the pgr and population density is strongly concave for many species, and hence populations spend most of their time at or above their carrying capacity. This conclusion is not supported statistically for any of the time series considered here. Although the local and global best-fit concave models may be appropriate for densities near the carrying capacity, they predict biologically implausible dynamics starting at low population densities (Table 2), and applications of such models could prove dangerous for conservation (Reynolds and Freckleton 2005, Staples and Taper 2006). The generality of both Sibly et al. (2005), and Sibly et al. (2007) which begins with "...1780 different populations found to be informative about the form of population regulation in the analysis of (Sibly et al. 2005)," needs to be reevaluated with analyses that at a minimum impose realistic phenomenological constraints and recognize the considerable uncertainty associated with point estimates for many populations. As in all population studies, care must be taken to both asses statistical certainty as discussed here and in Doncaster (2006), and, whenever

TABLE 2. Five-year prediction properties for 1000 simulated population time series using the models in Table 1 with different initial population sizes $N_{\text {init }}$.

\begin{tabular}{|c|c|c|c|c|c|c|c|c|c|}
\hline \multirow[b]{2}{*}{$\begin{array}{l}\text { Model and likelihood } \\
\text { surface location }\end{array}$} & \multicolumn{3}{|c|}{$N_{\text {init }}=0.1 \hat{N}^{*}$} & \multicolumn{3}{|c|}{$N_{\text {init }}=\hat{N}^{*}$} & \multicolumn{3}{|c|}{$N_{\text {init }}=4 \hat{N}^{*}$} \\
\hline & $\underset{(\%)}{\operatorname{Time}}$ & $\begin{array}{l}\text { Max. } \\
\text { population }\end{array}$ & Skewness & $\underset{(\%)}{\operatorname{Time}}$ & $\begin{array}{l}\text { Max. } \\
\text { population }\end{array}$ & Skewness & $\underset{(\%)}{\text { Time }}$ & $\begin{array}{l}\text { Max. } \\
\text { population }\end{array}$ & Skewness \\
\hline RPN, best fit & 15 & 45 & -0.09 & 39 & 46 & 0.05 & 29 & 137 & 0.95 \\
\hline TRPN, first quadrant best fit & 46 & 65 & -0.62 & 41 & 45 & 0.04 & 53 & 137 & 1.05 \\
\hline TRPN, third quadrant best fit & 80 & $4.4 \times 10^{200}$ & $\mathrm{ud} \dagger$ & 43 & 45 & 0.05 & 97 & 136 & 0.43 \\
\hline TRPN, ALT & 0 & 36 & 0.38 & 38 & 47 & 0.05 & 20 & 138 & 1.07 \\
\hline TRSS, first quadrant best fit & 44 & 63 & -0.30 & 40 & 40 & 0.02 & 56 & 137 & 1.03 \\
\hline TRSS, third quadrant best fit & 80 & inft & $\mathrm{ud} \dagger$ & 43 & 43 & 0.05 & 100 & 136 & 0.26 \\
\hline TRSS, ALT & 18 & 40 & 0.29 & 39 & 38 & -0.004 & 20 & 137 & 1.07 \\
\hline BHPN, best fit & 40 & 46 & -1.00 & 41 & 46 & 0.06 & 60 & 137 & 1.07 \\
\hline$\gamma$-BHPN, best fit & 46 & 59 & -0.73 & 40 & 46 & 0.06 & 53 & 137 & 1.05 \\
\hline$\gamma$-BHPN, ALT & 0 & 35 & 0.42 & 39 & 44 & 0.06 & 20 & 137 & 1.03 \\
\hline
\end{tabular}

Notes: Numbers correspond to the percentage of time spent above estimated carrying capacity, maximum population size, and mean sample skewness. Models are as in Table 1.

$\dagger$ Undefined.

$\$$ Infinite. 

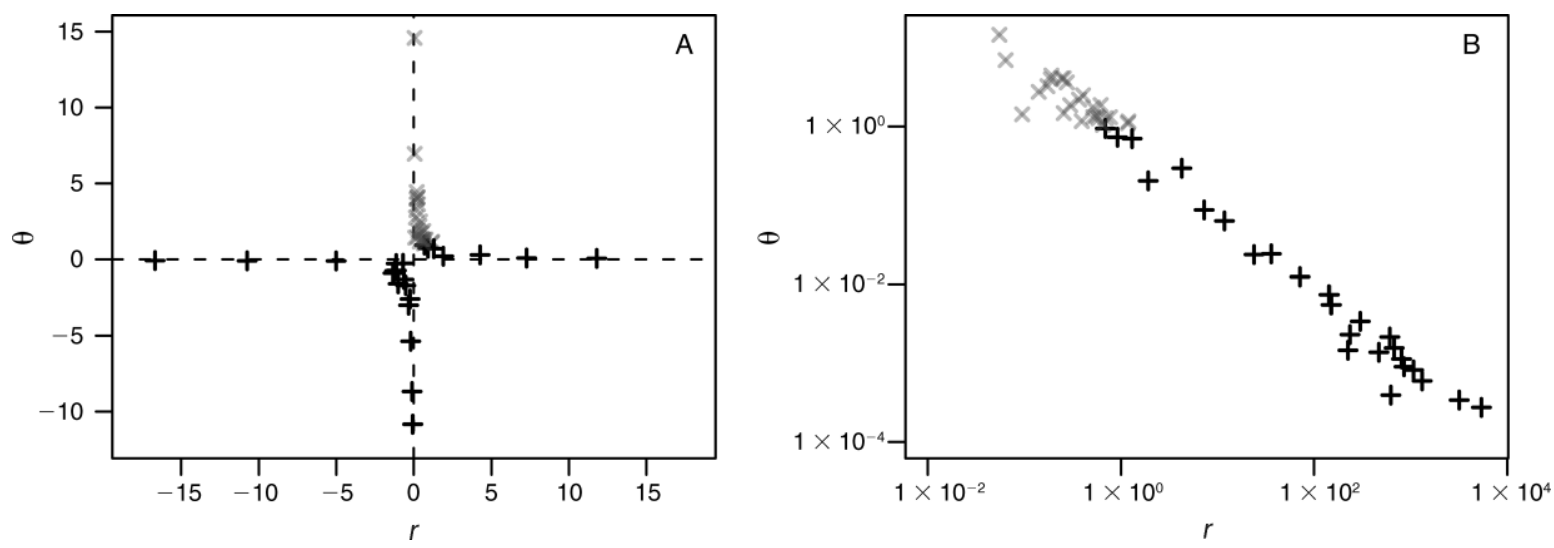

FIG. 3. Location of concave best-fit (denoted by black plus signs) and supported convex ALT (denoted by gray crosses) $r-\theta$ values from a theta-Ricker with process noise (TRPN) model fit to 25 data sets in which $r-\theta$ values were allowed to take on any values in panel (A) and bounded to biologically realistic values of the first quadrant in panel (B). Also see Appendix: Table A4 and Fig. A4.

possible, utilize other available information about the broader ecological system to ensure that the relatively simple models of Table 1 are appropriate (Getz and Lloyd-Smith 2006, Peacock and Garshelis 2006).

Likelihood ridges have implications for several other time series analysis approaches, where the common thread is the likelihood function. Information theoretic based methods of model comparison (Hurvich and Tsai 1991, Burnham and Anderson 2002, Bengtsson and Cavanaugh 2006) have been developed for time series models and represent a different paradigm from the hypothesis tests used for illustration here. Nevertheless, information criteria include maximum likelihoods for the "goodness-of-fit" term (the model complexity "penalty" remains unknown for nonlinear models), so all of our considerations are likely to translate directly. Attempts to incorporate model uncertainty using a set of candidate models such as those from Table 1 and an informationtheoretic based weighting scheme to improve predictions could be corrupted by biologically implausible models with nontrivial weights due to the comparable maximum likelihood values. Bayesian methods of data analysis also rely on the same likelihood functions as the key ingredient to update prior parameter distributions (West and Harrison 1989). Inferential statements about model parameters based on the posterior distribution of $\boldsymbol{\Theta}$, posterior predictive distributions of population states, and Bayesian model averaging all rely on the likelihood function to inform the model and subsequent predictions. Thus, because the ridge in the likelihood surface contains the best fitting model in realistic regions of parameter space, a strictly Bayesian approach would not be expected to improve the ambiguity about the "true" form of the pgr as a function of density, even after assigning zero mass to prior parameter distributions in regions describing implausible models (see also example studies by Ward [2006] and Sæther et al. [2007]). These cautions also apply to methods that utilize a Bayesian approach en rout to obtaining ML estimates (e.g., de Valpine 2004, Lele et al. 2007). Finally, Munch et al. (2005) have shown how nonparametric methods can offer a way to improve model performance in the absence of knowledge about the phenomenological form of density dependence. Given the improved realism of point estimates by our best-fit model incorporating observation error, we suggest research combining nonparametric and state-space models as useful direction for further research.

\section{ACKNOWLEDGMENTS}

This work was supported with funding to W. M. Getz from the James S. McDonnell Foundation and the NSF/NIH Evolution and Ecology of Infectious Disease Program. We thank several anonymous reviewers for helpful comments and the R Development Core Team.

\section{Literature Cited}

Aanes, S., S. Engen, B. E. Sæther, T. Willebrand, and V. Marcstrom. 2002. Sustainable harvesting strategies of Willow Ptarmigan in a fluctuating environment. Ecological Applications 12:281-290.

Bellows, T. S. 1981. The descriptive properties of some models for density dependence. Journal of Animal Ecology 50:139156.

Bengtsson, T., and J. E. Cavanaugh. 2006. An improved Akaike information criterion for state-space model selection. Computational Statistics and Data Analysis 50:2635-2654.

Burnham, K. P., and D. Anderson. 2002. Model selection and multimodel inference: a practical information-theoretic approach. Springer, New York, New York, USA.

Dennis, B., J. M. Ponciano, S. R. Lele, M. L. Taper, and D. F. Staples. 2006. Estimating density dependence, process noise, and observation error. Ecological Monographs 76:323-341.

Dennis, B., and M. L. Taper. 1994. Density dependence in timeseries observations of natural populations: estimation and testing. Ecological Monographs 64:205-224.

de Valpine, P. 2004. Monte Carlo state-space likelihoods by weighted posterior kernel density estimation. Journal of the American Statistical Association 99:523-536.

de Valpine, P., and A. Hastings. 2002. Fitting population models incorporating process noise and observation error. Ecological Monographs 72:57-76. 
de Valpine, P., and R. Hilborn. 2005. State-space likelihoods for nonlinear fisheries time-series. Canadian Journal of Fisheries and Aquatic Sciences 62:1937-1952.

Doncaster, C. P. 2006. Comment on "On the regulation of populations of mammals, birds, fish, and insects" III. Science 311:1100.

Ellner, S., and P. Turchin. 1995. Chaos in a noisy world: new methods and evidence from time-series analysis. American Naturalist 145:343-375.

Getz, W. M. 1996. A hypothesis regarding the abruptness of density dependence and the growth rate of populations. Ecology 77:2014-2026.

Getz, W. M., and R. G. Haight. 1989. Population harvesting: demographic models of fish, forest, and animal resources. Princeton University Press, Princeton, New Jersey, USA.

Getz, W. M., and J. O. Lloyd-Smith. 2006. Comment on "On the regulation of populations of mammals, birds, fish, and insects" I. Science 311:1100.

Ginzburg, L. R., S. Ferson, and H. R. Akcakaya. 1990. Reconstructibility of density dependence and the conservative assessment of extinction risks. Conservation Biology 4: 63-70.

Hilborn, R., and M. Mangel. 1997. The ecological detective: confronting models with data. Princeton University Press, Princeton, New Jersey, USA.

Hurvich, C. M., and C. L. Tsai. 1991. Bias of the corrected AIC criterion for underfitted regression and time-series models. Biometrika 78:499-509.

Kendall, B. E. 2001. Cycles, chaos, and noise in predator-prey dynamics. Chaos, Solitons and Fractals 12:321-332.

Krebs, C. J. 2002. Two complementary paradigms for analysing population dynamics. Philosophical Transactions of the Royal Society of London Series B 357:1211-1219.

Lele, S. R., B. Dennis, and F. Lutscher. 2007. Data cloning: easy maximum likelihood estimation for complex ecological models using Bayesian Markov chain Monte Carlo methods. Ecology Letters 10:551-563.

Lillegard, M., S. Engen, B. E. Sæther, V. Grotan, and M. C. Drever. 2008. Estimation of population parameters from aerial counts of North American mallards: a cautionary tale. Ecological Applications 18:197-207.

May, R. M., and G. F. Oster. 1976. Bifurcations and dynamic complexity in simple ecological models. American Naturalist 110:573-599.

Maynard Smith, J., and M. Slatkin. 1973. Stability of predatorprey systems. Ecology 54:384-391.

Meeker, W. Q., and L. A. Escobar. 1995. Teaching about approximate confidence-region based maximum-likelihoodestimation. American Statistician 49:48-53.

Munch, S. B., A. Kottas, and M. Mangel. 2005. Bayesian nonparametric analysis of stock-recruitment relationships. Canadian Journal of Fisheries and Aquatic Sciences 62:18081821.

Myers, R. A., K. G. Bowen, and N. J. Barrowman. 1999. Maximum reproductive rate of fish at low population sizes. Canadian Journal of Fisheries and Aquatic Sciences 56: 2404-2419.

Newton, I., and M. Marquiss. 1986. Population regulation in Sparrowhawks. Journal of Animal Ecology 55:463-480.

Newton, I., and P. Rothery. 1997. Senescence and reproductive value in Sparrowhawks. Ecology 78:1000-1008.
Peacock, E., and D. L. Garshelis. 2006. Comment on "On the regulation of populations of mammals, birds, fish, and insects" IV. Science 313:45.

Polansky, L., P. de Valpine, J. O. Lloyd-Smith, and W. M. Getz. 2008. Parameter estimation in a generalized discretetime model of density dependence. Theoretical Ecology 1: 221-229.

Reynolds, J. D., and R. P. Freckleton. 2005. Population dynamics: growing to extremes. Science 309:567-568.

Sæther, B. E., S. Engen, and R. Lande. 1996. Densitydependence and optimal harvesting of fluctuating populations. Oikos 76:40-46.

Sæther, B. E., S. Engen, R. Lande, P. Arcese, and J. N. M. Smith. 2000. Estimating the time to extinction in an island population of song sparrows. Proceedings of the Royal Society of London Series B 267:621-626.

Sæther, B. E., S. Engen, and E. Matthysen. 2002. Demographic characteristics and population dynamical patterns of solitary birds. Science 295:2070-2073.

Sæther, B. E., M. Lillegard, V. Grotan, F. Filli, and S. Engen. 2007. Predicting fluctuations of reintroduced ibex populations: the importance of density dependence, environmental stochasticity and uncertain population estimates. Journal of Animal Ecology 76:326-336.

Schaffer, W. M., S. Ellner, and M. Kot. 1986. Effects of noise on some dynamic-models in ecology. Journal of Mathematical Biology 24:479-523.

Severini, T. A. 2000. Likelihood methods in statistics. Oxford University Press, Oxford, UK.

Shumway, R. H., and D. S. Stoffer. 2000. Time series analysis and its applications. First edition. Springer-Verlag, Harrisonburg, Virginia, USA.

Sibly, R. M., D. Barker, M. C. Denham, J. Hone, and M. Pagel. 2005. On the regulation of populations of mammals, birds, fish, and insects. Science 309:607-610.

Sibly, R. M., D. Barker, J. Hone, and M. Pagel. 2007. On the stability of populations of mammals, birds, fish and insects. Ecology Letters 10:970-976.

Staples, D. F., and M. L. Taper. 2006. Impact of non-linearities in density dependence beyond the range of the data on predicting population risk. Journal of Nature Conservation 14:73-77.

Thomas, W. R., M. J. Pomerantz, and M. E. Gilpin. 1980. Chaos, asymmetric growth and group selection for dynamic stability. Ecology 61:1312-1320.

Turchin, P. 1990. Rarity of density dependence or population regulation with lags. Nature 344:660-663.

Turchin, P. 2003. Complex population dynamics: a theoretical/empirical synthesis. Princeton University Press, Princeton, New Jersey, USA.

Ward, E. J. 2006. A new BEAST: Bayesian software tools for ecological trend analysis. Wildlife Society Bulletin 34:1420 1424.

West, M., and J. Harrison. 1989. Bayesian forecasting and dynamic models. Springer-Verlag, New York, New York, USA.

Wolda, H., and B. Dennis. 1993. Density-dependence tests, are they. Oecologia 95:581-591.

Wood, S. N., and M. B. Thomas. 1999. Super-sensitivity to structure in biological models. Proceedings of the Royal Society B 266:565-570.

\section{APPENDIX}

Further discussion on the theta-Ricker and generalized Beverton-Holt density-dependent models, a complete description of likelihood equations and methods of parameter estimation, elaboration on the simulation experiment, additional analyses of the Accipiter nisus data set, TRPN best-fit and ALT models for 25 additional time series from the GPDD, and a presentation of the joint profile likelihood surface of a Columba oenas data set for comparison with Bayesian posterior densities (Ecological Archives E090-161-A1). 